\title{
PANCYCLICITY WHEN EACH CYCLE MUST PASS EXACTLY $k$ HAMILTON CYCLE CHORDS
}

\author{
Fatima Affif Chaouche \\ University of Sciences and \\ Technology Houari Boumediene \\ Algiers, Algeria \\ e-mail: f_affif@yahoo.fr \\ CARrie G. Rutherford \\ Faculty of Business \\ London South Bank University \\ London, UK \\ e-mail: c.g.rutherford@lsbu.ac.uk
}

AND

RoBin W. WhitTY

School of Mathematical Sciences

Queen Mary University of London

London, UK

e-mail: r.whitty@qmul.ac.uk

\begin{abstract}
It is known that $\Theta(\log n)$ chords must be added to an $n$-cycle to produce a pancyclic graph; for vertex pancyclicity, where every vertex belongs to a cycle of every length, $\Theta(n)$ chords are required. A possibly 'intermediate' variation is the following: given $k, 1 \leq k \leq n$, how many chords must be added to ensure that there exist cycles of every possible length each of which passes exactly $k$ chords? For fixed $k$, we establish a lower bound of $\Omega\left(n^{1 / k}\right)$ on the growth rate.
\end{abstract}

Keywords: extremal graph theory, pancyclic graph, Hamilton cycle.

2010 Mathematics Subject Classification: 05C38. 
[1] J.A. Bondy, Pancyclic graphs I, J. Combin. Theory Ser. B 11 (1971) 80-84. doi:10.1016/0095-8956(71)90016-5

[2] J.A. Bondy, Pancyclic graphs: recent results, infinite and finite sets, in : Colloq. Math. Soc. János Bolyai, Keszthely, Hungary (1973) 181-187.

[3] H.J. Broersma, A note on the minimum size of a vertex pancyclic graph, Discrete Math. 164 (1997) 29-32. doi:10.1016/S0012-365X(96)00040-4

[4] R.M. Corless, G.H. Gonnet, D.E.G. Hare, D.J. Jeffrey and D.E. Knuth, On the Lambert $W$ function, Adv. Comput. Math. 5 (1996) 329-359. doi:10.1007/BF02124750

[5] J.C. George, A.M. Marr and W.D. Wallis, Minimal pancyclic graphs, J. Combin. Math. Combin. Comput. 86 (2013) 125-133.

[6] S. Griffin, Minimal pancyclicity, preprint, arxiv.org/abs/1312.0274, 2013.

[7] M.R. Sridharan, On an extremal problem concerning pancyclic graphs, J. Math. Phys. Sci. 12 (1978) 297-306.

Received 14 April 2014

Revised 7 November 2014

Accepted 7 November 2014 